\title{
Martensitic Phase Transformations in Quaternary Heusler Alloys $\mathrm{Co}_{2} \mathrm{Ti}_{1-x} \mathrm{Fe}_{x} \mathrm{As}$
}

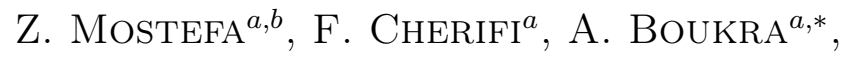 \\ Z.F. MeghoufeL ${ }^{a}$ AND A. Boukortt ${ }^{b}$ \\ ${ }^{a}$ Laboratoire de Structure, Elaboration et Application des Matériaux Moléculaires, \\ SEA2M, Université Abdelhamid Ibn Badis, Chemin des Crêtes, 27000, Mostaganem, Algeria \\ ${ }^{b}$ Laboratoire d'Elaboration et Caractérisation Physico-Mécanique \\ et Métallurgique des Matériaux, ECP3M, Université Abdelhamid Ibn Badis, \\ Route Belhacel, 27000, Mostaganem, Algeria
}

Received: 23.03.2021 \& Accepted: 30.06.2021

Doi: $10.12693 /$ APhysPolA.140.175

*e-mail: abdelaziz.boukra@univ-mosta.dz

\begin{abstract}
$A b$ initio calculations are used to predict tetragonal transformation of $\mathrm{Co}_{2} \mathrm{Ti}_{1-x} \mathrm{Fe}_{x}$ As Heusler alloys. We show that distortion of the cubic (austenite) Heusler structure induces a tetragonal (martensite) structure with space group $I 4 / \mathrm{mmm}$. At tetragonality, with the $\mathrm{c} / a$ ratio of $1.30,1.26,1.22,1.17$, and 1.16 corresponding to $x=0,0.25,0.5,0.75$, and 1 , respectively, the martensite phases become energetically more stable than the austenite ones. The negative values of tetragonal shear modulus parameter mean that the austenite phases of these alloys are elastically unstable, and consequently are expected to undergo martensitic transformations. The stability of the martensitic phases is confirmed by calculating the total spin polarized density of states at the Fermi level. The addition of Fe to the $\mathrm{Co}_{2}$ TiAs alloy decreases the energy difference between the austenite and martensite phases, leading to a decrease of the martensitic transformation temperature $T_{M}$. With $T_{M}$ higher than the room temperature for $x=0$ and 0.25 , these compounds are expected to behave like high-temperature shape memory alloys.
\end{abstract}

topics: martensite phase, austenite phase, martensitic transformation temperature, shape memory alloy

\section{Introduction}

Full Heusler alloys are ternary compounds with the $\mathrm{X}_{2} \mathrm{YZ}$ formula, where $\mathrm{X}$ and $\mathrm{Y}$ are transition metals and $\mathrm{Z}$ is the main group element. They crystallize in a $\mathrm{Cu}_{2} \mathrm{MnAl}$ cubic structure with space group $F m \overline{3} m$. The $\mathrm{X}$ atoms occupy $(1 / 4,1 / 4,1 / 4)$ and $(3 / 4,3 / 4,3 / 4)$ positions while the $\mathrm{Y}$ and $\mathrm{Z}$ atoms are located on $(1 / 2,1 / 2,1 / 2)$ and $(0,0,0)$ positions, respectively. The Heusler alloys present a vast variety of properties, ranging from semiconductors, half-metallic ferromagnets, superconductors, and topological insulators to shape memory alloys. They are considered potential candidates for various practical applications, including spintronic [1], thermoelectric [2] and magnetic refrigeration or magnetostrictive transducers [3, 4]. Shape memory alloys (SMAs) have the stunning property of recovering their initial shape after deformation which can be induced by submitting to a change in temperature or stress. A solid-solid structural transformation from the parent phase (austenite) to the product (martensite) phase occurs at the martensitic transformation temperature $T_{M}$ (martensite start temperature). However, industrial SMAs have a low operating temperature drawback, which reduces their potential applications. This is the reason why there is a technological need to develop new SMAs with high $T_{M}$, which will open up unexplored application fields.

Ferromagnetic shape memory alloys (FSMA) are a subgroup of shape memory alloys (SMA) that also exhibit a ferromagnetic behavior. In this way, the $\mathrm{Ni}_{2} \mathrm{MnGa}$ compound, one of the best understood FSMA, has been extensively studied [5-11]. It undergoes a transition from a cubic to a tetragonal distorted structure at $T_{M}$ around $200 \mathrm{~K}$, which could be enhanced by changing the concentration of the Ni-Mn-Ga alloy's constituent elements, leading to $T_{M}$ equal to $330 \mathrm{~K}$ [12]. The FSMA has also been observed in the Heusler compounds containing $\mathrm{Co}$ and $\mathrm{Ni}$ atoms, such as $\mathrm{Co}_{2} \mathrm{NiGa}$ and $\mathrm{Co}_{2} \mathrm{NiAl}$ [13-17]. The studies carried out on $\mathrm{Ni}-\mathrm{Fe}-\mathrm{Co}-\mathrm{Mn}-\mathrm{Sn}$ alloys by $\mathrm{Qu}$ et al. [18] have led to results of great importance for the design of high performance multifunctional materials. Moreover, Chen et al. have observed experimentally that the $\mathrm{NiCoFeGa}$ alloy system with various Co contents deformed in tension at various temperatures displays a supercritical elasticity [19]. On the one 
hand, in the Co-Ti-based Heusler alloys, the majority of theoretical and experimental researches have focused on magnetic, optical and electronic transport properties. In this view, we cite magnetic, half-metallic and optical properties of the full-Heusler alloys $\mathrm{Co}_{2} \mathrm{TiX}(\mathrm{X}=\mathrm{Al}, \mathrm{Ga}, \mathrm{Si}, \mathrm{Ge}$, $\mathrm{Sn}, \mathrm{Sb})$ carried out theoretically and experimentally by Lee et al., Webster and Ziebeck, Bainsla and Suresh, and Shreder et al. [20-23], magnetic and transport properties of $\mathrm{Co}_{2} \mathrm{Ti}_{1-x} \mathrm{Fe}_{x}$ Ge studied experimentally by Venkateswarlu et al. [24], electronic and magnetic properties of the full-Heusler alloys $\mathrm{Co}_{2} \mathrm{Ti}_{1-x} \mathrm{Fe}_{x}$ Ga studied theoretically by Ahmadian and Boochani [25] and last but not least, half-metallic ferromagnetic behavior observed experimentally in $\mathrm{Co}_{2} \mathrm{Ti}_{1-x} \mathrm{Fe}_{x} \mathrm{Sn}$ by Rani et al. [26] On the other hand, the martensitic transformation has been experimentally observed and theoretically predicted in the $\mathrm{Co}_{2} \mathrm{TiSb}_{1-x} \mathrm{Sn}_{x}$ alloy by Wang et al. [27, 28]. The main concern of the present work is to provide theoretical prediction about martensitic transformations in $\mathrm{Co}_{2} \mathrm{Ti}_{1-x} \mathrm{Fe}_{x}$ As $(x=0$, $0.25,0.5,0.75,1)$ alloys.

\section{Calculation details}

The calculations are carried out within density functional theory. We use the all-electron full potential linearized augmented plane-wave method as implemented in the Wien $2 \mathrm{k}$ code [29]. The exchange and correlation effects are introduced using the generalized gradient approximation (GGA) based on the Perdew-Burke-Ernzerhof (PBE) approach [30]. We use a 12-atom supercell, corresponding to a $1 \times 1 \times 1$ conventional cubic cell. The cubic and tetragonal crystal structures of the $\mathrm{Co}_{2}$ TiAs alloy visualized by the Xcrysden tool [31] are shown in Fig. 1.

In the cubic Heusler structure (space group $F m \overline{3} m)$, the Co atoms occupy $8 \mathrm{c}(1 / 4,1 / 4,1 / 4)$ positions, the $\mathrm{Ti}$ and $\mathrm{As}$ atoms are located at the Wyckoff position of $4 \mathrm{~b}(1 / 2,1 / 2,1 / 2)$ and $4 \mathrm{a}$ $(0,0,0)$, respectively. The calculations are performed for the $\mathrm{Co}_{2} \mathrm{Ti}_{1-x} \mathrm{Fe}_{x}$ As alloys with $5 \mathrm{Fe}$ compositions $(x=0,0.25,0.50,0.75$ and 1$)$. For $x=0.25$, one Fe atom substitutes one $\mathrm{Ti}$ atom

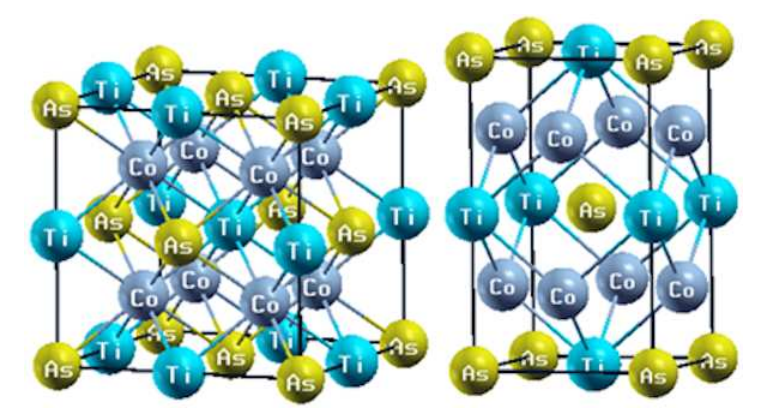

Fig. 1. Crystal structures of the $\mathrm{Co}_{2} \mathrm{TiAs}$ alloy, cubic (left) and tetragonal (right). at $(1 / 2,0,0)$ position. For $x=0.50$, two Ti atoms are replaced by two Fe atoms at positions $(1 / 2,0,0)$ and $(0,1 / 2,0)$, respectively. For $x=0.75$, three $\mathrm{Ti}$ atoms are substituted by three Fe atoms at positions $(1 / 2,0,0),(0,1 / 2,0)$ and $(0,0,1 / 2)$, respectively. For $x=1$, all four Ti atoms are replaced by four Fe atoms. In the tetragonal structure with space group $I 4 / \mathrm{mmm}$, the Co atoms occupy the Wyckoff position $4 \mathrm{~d}(0,1 / 2,1 / 4)$, the Ti and the As atoms are located at $2 \mathrm{~b}(0,0,1 / 2)$ and $2 \mathrm{a}(0,0,0)$, respectively.

We expand the basis function up to $R_{M T} K_{\max }=7 \quad\left(R_{M T}\right.$ is the muffin-tin radius and $K_{\max }$ is the maximum modulus for reciprocal lattice vectors). The $R_{M T}$ spheres adopted are 2.1, 2.0, 2.1, and 2.2 a.u. (atomic units) for Co, $\mathrm{Ti}, \mathrm{Fe}$, and As atoms, respectively. The Brillouin zone integrations were performed with the special $k$-point method of the Monkhorst-Pack mesh [32] over a $9 \times 9 \times 9$ mesh. Self-consistency was achieved with an energy tolerance of $10^{-4} \mathrm{Ry}$. The elastic constants are calculated using the thermo_pw package [33] integrated in the Quantum ESPRESSO code [34]. The PBE-GGA is used for the exchange-correlation functional with ultra-soft pseudopotentials.

\section{Results}

\subsection{Structural and mechanical properties}

The total energy for cubic and tetragonal Heusler structures of $\mathrm{Co}_{2} \mathrm{Ti}_{1-x} \mathrm{Fe}_{x}$ As alloys in the ferromagnetic state have been calculated for different volumes and fitted by the Murnaghan equation of state [35]. The corresponding equilibrium lattice parameter $a$, bulk modulus $B$ and its pressure derivative $B^{\prime}$ are summarized in Table I. We note that experimental and theoretical lattice constants of these alloys are still unavailable to be compared.

At the equilibrium state, the variation of calculated lattice parameters of the cubic phase versus the Fe composition obeys Vegard's law [36] as shown in Fig. 2.

TABLE I

Calculated structural properties of $\mathrm{Co}_{2} \mathrm{Ti}_{1-x} \mathrm{Fe}_{x} \mathrm{As}$ alloys in the cubic and tetragonal phases.

\begin{tabular}{c|l|c|c|c|c}
\hline \hline \multicolumn{1}{c|}{ Compound } & \multicolumn{1}{|c|}{ Phase } & $a[\AA]$ & $c / a$ & $B[\mathrm{GPa}]$ & $B^{\prime}$ \\
\hline $\mathrm{Co}_{2} \mathrm{TiAs}$ & cubic & 5.860 & 1 & 163.38 & 4.46 \\
& tetragonal & 5.335 & 1.30 & 182.56 & 4.44 \\
\hline \multirow{2}{*}{$\mathrm{Co}_{2} \mathrm{Ti}_{0.75} \mathrm{Fe}_{0.25} \mathrm{As}$} & cubic & 5.838 & 1 & 167.55 & 4.42 \\
& tetragonal & 5.374 & 1.26 & 175.39 & 4.52 \\
\hline \multirow{2}{*}{$\mathrm{Co}_{2} \mathrm{Ti}_{0.5} \mathrm{Fe}_{0.5} \mathrm{As}$} & cubic & 5.822 & 1 & 163.99 & 4.39 \\
& tetragonal & 5.423 & 1.22 & 166.533 & 4.06 \\
\hline \multirow{2}{*}{$\mathrm{Co}_{2} \mathrm{Ti}_{0.25} \mathrm{Fe}_{0.75} \mathrm{As}$} & cubic & 5.804 & 1 & 160.61 & 4.48 \\
& tetragonal & 5.462 & 1.17 & 168.75 & 4.53 \\
\hline \multirow{2}{*}{$\mathrm{Co}_{2} \mathrm{FeAs}$} & cubic & 5.777 & 1 & 162.19 & 4.06 \\
& tetragonal & 5.433 & 1.16 & 167.43 & 4.49
\end{tabular}


TABLE II

Calculated mechanical properties and martensite transformation temperature of $\mathrm{Co}_{2} \mathrm{Ti}_{1-x} \mathrm{Fe}_{x} \mathrm{As}$ alloys.

\begin{tabular}{|c|c|c|c|c|c|c|c|c|c|}
\hline Compound & Phase & $\begin{array}{c}C_{11} \\
{[\mathrm{GPa}]}\end{array}$ & $\begin{array}{c}C_{12} \\
{[\mathrm{GPa}]}\end{array}$ & $\begin{array}{c}C_{13} \\
{[\mathrm{GPa}]}\end{array}$ & $\begin{array}{c}C_{33} \\
{[\mathrm{GPa}]}\end{array}$ & $\begin{array}{c}C_{44} \\
{[\mathrm{GPa}]}\end{array}$ & $\begin{array}{c}C_{66} \\
{[\mathrm{GPa}]}\end{array}$ & $\begin{array}{c}C^{\prime} \\
{[\mathrm{GPa}]}\end{array}$ & $\begin{array}{l}T_{M} \\
{[\mathrm{~K}]}\end{array}$ \\
\hline \multirow{2}{*}{$\mathrm{Co}_{2} \mathrm{TiAs}$} & cubic & 77.32 & 215.37 & - & - & 63.01 & - & -69.02 & \multirow{2}{*}{998.31} \\
\hline & tetragonal & 284.51 & 103.56 & 177.03 & 224.15 & 51.19 & 42.6 & 90.47 & \\
\hline \multirow{2}{*}{$\mathrm{Co}_{2} \mathrm{Ti}_{0.75} \mathrm{Fe}_{0.25} \mathrm{As}$} & cubic & 88.73 & 208.43 & - & - & 62.56 & - & -59.84 & \multirow{2}{*}{652.38} \\
\hline & tetragonal & 260.39 & 103.10 & 175.05 & 224.17 & 55.82 & 46.19 & 78.64 & \\
\hline \multirow{2}{*}{$\mathrm{Co}_{2} \mathrm{Ti}_{0.5} \mathrm{Fe}_{0.5} \mathrm{As}$} & cubic & 100.42 & 197.98 & - & - & 62.41 & - & -48.77 & \multirow{2}{*}{267.73} \\
\hline & tetragonal & 244.54 & 102.66 & 172.17 & 224.22 & 59.61 & 50.45 & 70.74 & \\
\hline \multirow{2}{*}{$\mathrm{Co}_{2} \mathrm{Ti}_{0.25} \mathrm{Fe}_{0.75} \mathrm{As}$} & cubic & 111.03 & 190.50 & - & - & 62.31 & - & -39.73 & \multirow{2}{*}{193.49} \\
\hline & tetragonal & 236.95 & 103.09 & 168.37 & 235.57 & 62.55 & 56.49 & 66.93 & \\
\hline \multirow{2}{*}{$\mathrm{Co}_{2} \mathrm{FeAs}$} & cubic & 124.06 & 190.34 & - & - & 66.89 & - & -33.14 & \multirow{2}{*}{28.60} \\
\hline & tetragonal & 237.73 & 103.56 & 163.68 & 247.48 & 64.64 & 63.50 & 67.08 & \\
\hline
\end{tabular}

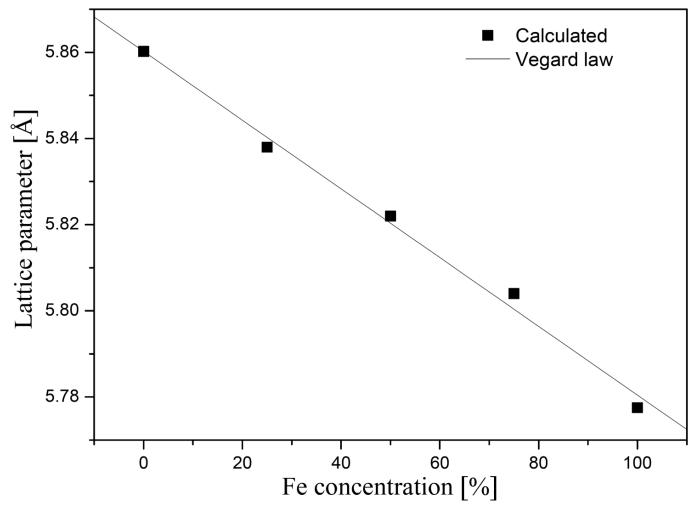

Fig. 2. Calculated lattice parameters versus $\mathrm{Fe}$ composition of the cubic phase together with Vegard's law variation of $\mathrm{Co}_{2} \mathrm{Ti}_{1-x} \mathrm{Fe}_{x}$ As alloys.

The mechanical stability of $\mathrm{Co}_{2} \mathrm{Ti}_{1-x} \mathrm{Fe}_{x}$ As alloys is estimated by calculating the elastic constants $C_{i j}$. In the tetragonal lattice symmetry, there are six independent elastic constants $C_{11}, C_{12}, C_{13}$, $C_{33}, C_{44}$, and $C_{66}$, which are reduced to three $\left(C_{11}\right.$, $C_{12}$, and $\left.C_{44}\right)$ in the cubic symmetry case. The calculated constants of all studied alloys in both symmetries are summarized in Table II. The Born mechanical stability criteria [37] for a cubic crystal requires that $C_{11}-C_{12}>0, C_{11}>0, C_{11}+2 C_{12}>0$ and $C_{44}>0$ (for a tetragonal crystal, $C_{11}-C_{12}>0$, $C_{33}>0, C_{44}>0, C_{66}>0, C_{11}+C_{33}-2 C_{13}>0$ and $\left.2 C_{11}+C_{33}+2 C_{12}+4 C_{13}>0\right)$. From Table II, it is clear that the elastic constants of tetragonal structures satisfy all the conditions, whereas those of cubic ones are not fulfilled, which indicates that these alloys are mechanically unstable in the cubic phase and are expected to undergo martensitic transformations. This is confirmed by the negative values of the tetragonal shear modulus parameter, $C^{\prime}=\left(C_{11}-C_{12}\right) / 2$, in the austenite phases (see Table II). We notice that $C^{\prime}$ of tetragonal martensitic phases decreases and that of cubic austenite phases increases, with increasing Fe concentration.

\subsection{Prediction of martensitic transformation}

To study a structural transformation from the cubic austenite phase to the tetragonal martensite phase, we calculate the total energy versus the tetragonal distortion, by varying the $c / a$ ratio and keeping the volume unchanged. Distortions of the cubic Heusler structures induce tetragonal structures with space group $I 4 / \mathrm{mmm}$. The energy difference between the martensite and austenite phases versus $c / a$ deformations of $\mathrm{Co}_{2} \mathrm{Ti}_{1-x} \mathrm{Fe}_{x} \mathrm{As}$ Heusler alloys are shown in Fig. 3. The results witness that, for each concentration, there are two energy minima, a shallow one at $c / a<1$ and a deeper one at $c / a>1$. The tetragonally distorted structures are energetically favorable to the cubic ones with a local energy minima of $c / a=1.30,1.26$, $1.22,1.17$, and 1.16 for $x=0,0.25,0.5,0.75$, and 1 , respectively. This indicates that at temperature change, a transformation from the austenite to the martensite phase may happen in $\mathrm{Co}_{2} \mathrm{Ti}_{1-x} \mathrm{Fe}_{x} \mathrm{As}$ Heusler alloys.

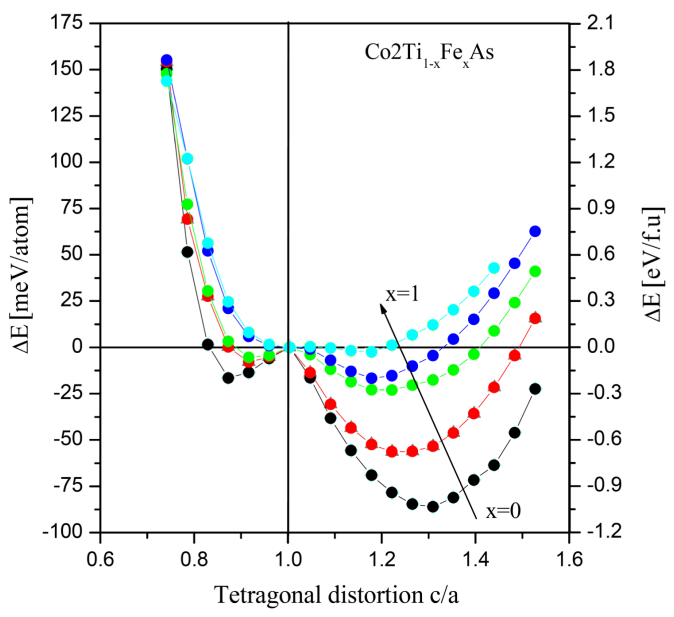

Fig. 3. Energy difference, $\Delta E$, between the martensite and austenite phases versus tetragonal distortion $c / a$ of $\mathrm{Co}_{2} \mathrm{Ti}_{1-x} \mathrm{Fe}_{x}$ As alloys. 


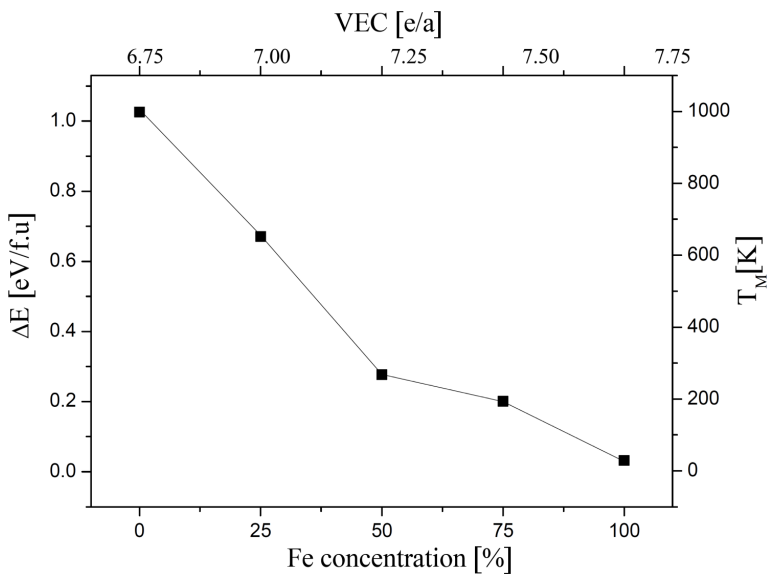

Fig. 4. Energy difference between the austenite and martensite phases, $\Delta E$, versus Fe composition of $\mathrm{Co}_{2} \mathrm{Ti}_{1-x} \mathrm{Fe}_{x}$ As alloys.

It has been reported that a large difference in stabilization energy, $\Delta E$, which is defined as the energy difference between the austenite and the martensite phases, implies a high stability of the martensitic phase and a change in the martensitic transformation temperature $T_{M}$ [38]. We find that, when the lattice is deformed, the total energy of the tetragonal phase is reduced by $86.06,56.24,23.08$, 16.70 , and $2.46 \mathrm{meV} /$ atom for $x=0,0.25,0.50$, 0.75 , and 1 , respectively. By comparing the calculated energy difference and the experimentally measured martensitic transformation temperature for several compounds, Barman et al. [38] found an approximately linear relationship between $\Delta E$ and $T_{M}, \Delta E \propto k_{\mathrm{B}} T_{M}$, where $k_{\mathrm{B}}$ is the Boltzmann constant. According to Fig. $4, \Delta E$ decreases with increasing $\mathrm{Fe}$ concentration and consequently $T_{M}$ decreases. For $x$ ranging from 0 to 1 , which corresponds to an increase of valence electron concentration per atom (e/a) from 6.75 to $7.75, T_{M}$ decreases from 998 to $28 \mathrm{~K}$. The e/a number is closely related to $T_{M}$ in a large variety of Heusler alloys [39-41]. Table II demonstrates that $\mathrm{Co}_{2} \mathrm{Ti}_{1-x} \mathrm{Fe}_{x}$ As alloys follow the general rule regarding the tetragonal shear modulus $C^{\prime}$ and $T_{M}$, a smaller $C^{\prime}$ indicates a higher $T_{M}[42-45]$.

To confirm the stability of the tetragonal phase with respect to the cubic one, we plot in Fig. 5 the total spin polarized density of states (DOS) at the Fermi level $\left(E_{\mathrm{F}}\right)$ for the martensite and austenite phases of $\mathrm{Co}_{2} \mathrm{Ti}_{1-x} \mathrm{Fe}_{x}$ As alloys. In the cubic phases, the calculated total DOSs of the studied compounds reveal the existence of peaks near the Fermi level $E_{\mathrm{F}}$. Faleev et al. [46], after analyzing 286 Heusler compounds, concluded that the main reason of the tetragonal distortion of many Heusler compounds is the existence of DOS peaks near $E_{\mathrm{F}}$ in the cubic phases. Figure 5 shows that the tetragonal distortion reduces the minority spins (spin-down) DOS at $E_{\mathrm{F}}$, while the majority spins (spin-up) DOS at $E_{\mathrm{F}}$ remains almost unchanged. Indeed, it has

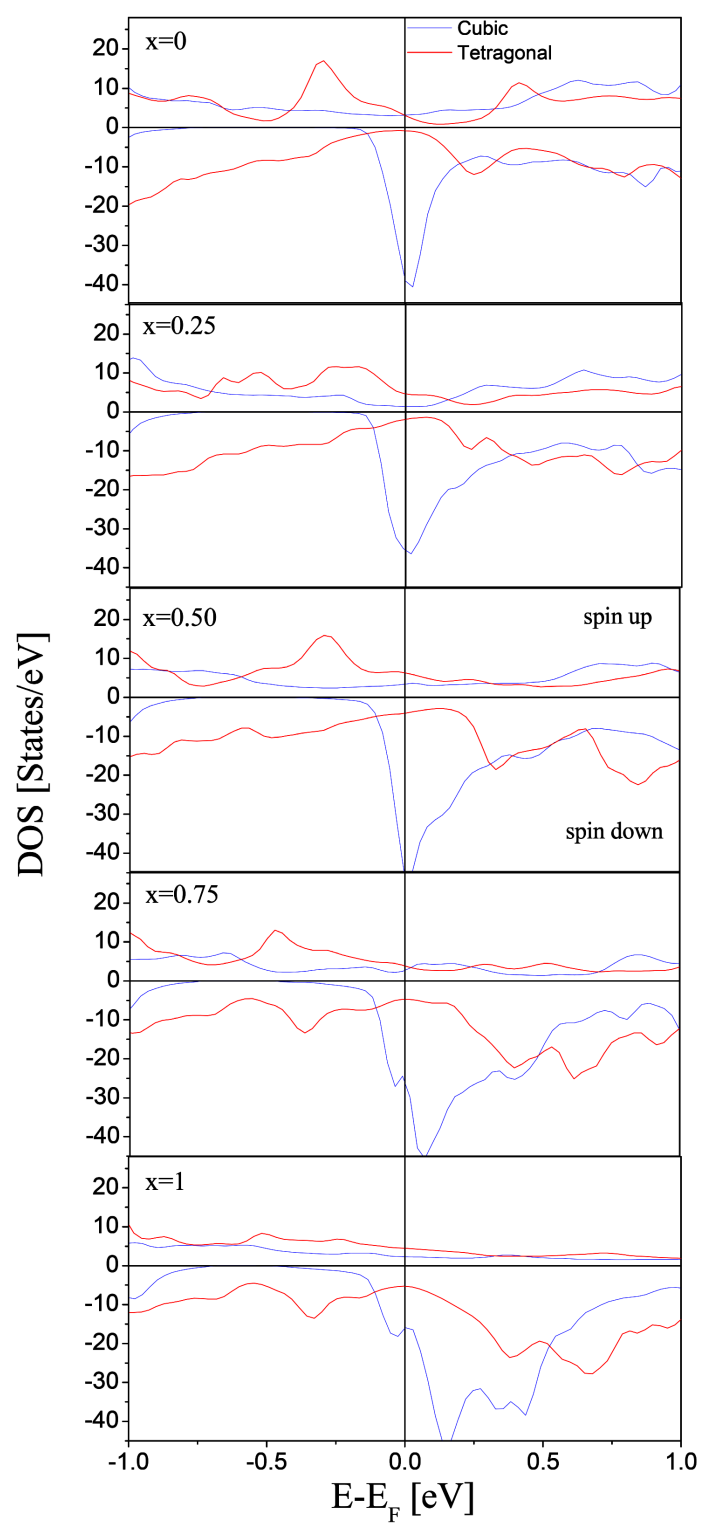

Fig. 5. DOS for the austenite and martensite phases of $\mathrm{Co}_{2} \mathrm{Ti}_{1-x} \mathrm{Fe}_{x}$ As alloys.

been reported by $\mathrm{Xu}$ and Oguchi [47] that high DOS at the Fermi level reduces the structure stability. Thus, we conclude that the tetragonal phases are most stable.

To measure the asymmetry of the DOS at $E_{\mathrm{F}}$ for spin up, $N^{\uparrow}\left(E_{\mathrm{F}}\right)$, and spin down, $N^{\downarrow}\left(E_{\mathrm{F}}\right)$, electrons, we calculate the spin polarization $(P)$ at $E_{\mathrm{F}}$ with

$$
P=\frac{N^{\uparrow}\left(E_{\mathrm{F}}\right)-N^{\downarrow}\left(E_{\mathrm{F}}\right)}{N^{\uparrow}\left(E_{\mathrm{F}}\right)+N^{\downarrow}\left(E_{\mathrm{F}}\right)} .
$$

The polarization decreases as a function of the concentration of $\mathrm{Fe}$ in both the cubic and tetragonal phases, and it is found equal to $84,92,86,81$, and $74 \%$ in the cubic phases and $58,41,21,7$, and $8 \%$ in the tetragonal ones, estimated at concentrations $x=25,50,75$, and $100 \%$, respectively. We notice that the tetragonal distortion reduces the spin polarization of the cubic parent phases. 


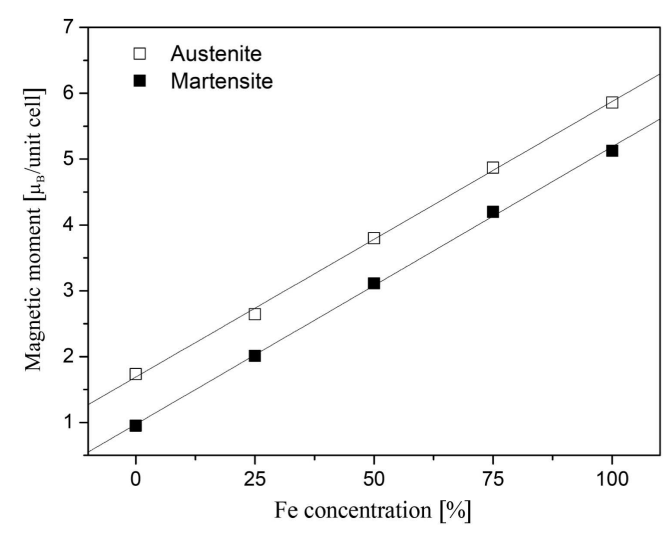

Fig. 6. Total magnetic moment versus Fe composition for the austenite and martensite phases of $\mathrm{Co}_{2} \mathrm{Ti}_{1-x} \mathrm{Fe}_{x}$ As alloys.

TABLE III

Calculated atomic magnetic moment (in $\mu_{\mathrm{B}}$ /atom) of $\mathrm{Co}_{2} \mathrm{Ti}_{1-x} \mathrm{Fe}_{x}$ As alloys.

\begin{tabular}{l|l|c|c|c|c}
\hline \hline Compound & Phase & $\mathrm{Co}$ & $\mathrm{Ti}$ & $\mathrm{Fe}$ & $\mathrm{As}$ \\
\hline \multirow{2}{*}{$\mathrm{Co}_{2} \mathrm{TiAs}$} & cubic & 0.87 & -0.004 & - & 0.04 \\
& tetragonal & 0.53 & -0.07 & - & 0.03 \\
\hline \multirow{2}{*}{$\mathrm{Co}_{2} \mathrm{Ti}_{0.75} \mathrm{Fe}_{0.25} \mathrm{As}$} & cubic & 0.97 & -0.05 & 2.84 & 0.07 \\
& tetragonal & 0.71 & -0.11 & 2.85 & 0.03 \\
\hline \multirow{2}{*}{$\mathrm{Co}_{2} \mathrm{Ti}_{0.5} \mathrm{Fe}_{0.5} \mathrm{As}$} & cubic & 1.19 & -0.07 & 2.85 & 0.08 \\
& tetragonal & 0.90 & -0.15 & 2.83 & 0.04 \\
\hline \multirow{2}{*}{$\mathrm{Co}_{2} \mathrm{Ti}_{0.25} \mathrm{Fe}_{0.75} \mathrm{As}$} & cubic & 1.36 & -0.04 & 2.81 & 0.06 \\
& tetragonal & 1.06 & -0.14 & 2.81 & 0.05 \\
\hline \multirow{2}{*}{$\mathrm{Co}_{2} \mathrm{FeAs}$} & cubic & 1.49 & - & 2.81 & 0.07 \\
& tetragonal & 1.17 & - & 2.77 & 0.03
\end{tabular}

This incomplete spin polarization at $E_{\mathrm{F}}$ gives rise to an incomplete half-metallicity and thus we expect non-integer values of the total magnetic moments.

Figure 6 represents the total magnetic moment of the primitive unit cell versus the Fe concentration. The addition of $\mathrm{Fe}$ to $\mathrm{Co}_{2} \mathrm{TiAs}$ increases linearly the magnetization in both the martensite and austenite phases. We have seen that the tetragonal distortion reduces the minority spins (spin-down) DOS at $E_{\mathrm{F}}$, and this is due to a charge transfer from the occupied to the unoccupied states. This transfer induces holes in the minority $d$-states and therefore reduces the total magnetic moment of the martensite phase. The atomic magnetic moments for the cubic and tetragonal $\mathrm{Co}_{2} \mathrm{Ti}_{1-x} \mathrm{Fe}_{x} \mathrm{As}(x=0,0.25,0.5,0.75$, and 1) alloys are listed in Table III. We notice that the atomic magnetic moments change slightly with lattice distortion. The $\mathrm{Ti}$ and As atoms show the smallest magnetic moment, thus the magnetization for these alloys is mainly due to the $\mathrm{Fe}$ and $\mathrm{Co}$ atoms.

\section{Conclusion}

We predict by means of first-principles calculations that tetragonal distortions of $\mathrm{Co}_{2} \mathrm{Ti}_{1-x} \mathrm{Fe}_{x} \mathrm{As}$ cubic alloys induce martensite phase transformations. The stability of the martensitic phase of these alloys is confirmed by the calculations of the total energy difference $\Delta E$ between the austenitic and martensitic phases, the tetragonal shear modulus $C^{\prime}$ and the total spin polarized density of the states at the Fermi level. We observe that $\Delta E$ decreases with increasing $\mathrm{Fe}$ composition resulting into the change of the martensite transformation temperature $T_{M}$ from 998 to $28 \mathrm{~K}$.

\section{References}

[1] Spintronics: From Materials to Devices, Eds. C. Felser, G.H. Fecher, Springer, Netherlands 2013.

[2] J. Barth, G.H. Fecher, B. Balke, T. Graf, C. Felser, A. Shkabko, A. Weidenkaff, Phys. Rev. B 81, 1 (2010).

[3] X. Zhou, W. Li, H.P. Kunkel, G. Williams, J. Phys. Condens. Matter 16, L39 (2004).

[4] S.J. Murray, M. Marioni, S.M. Allen, R.C. O'Handley, T.A. Lograsso, Appl. Phys. Lett. 77, 886 (2000).

[5] K. Ullakko, J.K. Huang, C. Kantner, R.C. O'Handley, V.V. Kokorin, Appl. Phys. Lett. 69, 1966 (1996).

[6] K. Ooiwa, K. Endo, A. Shinogi, J. Magn. Magn. Mater. 104-107, 2011 (1992).

[7] V.A. Chernenko, E. Cesari, V.V. Kokorin, I.N. Vitenko, Scr. Mater. 33, 1239 (1995).

[8] R. Tickle, R.D. James, J. Magn. Magn. Mater. 195, 627 (1999).

[9] M. Ohtsuka, M. Sanada, M. Matsumoto, K. Itagaki, Mater. Sci. Eng. A 378, 377 (2004).

[10] V.A. Chernenko, S. Besseghini, T. Kanomata, H. Yoshidad, T. Kakeshita, Scr. Mater. 55, 303 (2006).

[11] S. Özdemir Kart, M. Uludoğan, I. Karaman, T. Çă̆ın, Phys. Status Solidi A 205, 1026 (2008).

[12] T. Sakon, K. Sasaki, D. Numakura, M. Abe, H. Nojiri, Y. Adachi, T. Kanomata, Mater. Trans. 54, 9 (2013).

[13] C. Craciunescu, Y. Kishi, T.A. Lograsso, M. Wuttig, Scr. Mater. 47, 285 (2002).

[14] M. Sato, T. Okazaki, Y. Furuya, M. Wuttig, Mater. Trans. 44, 372 (2003).

[15] M. Sato, T. Okazaki, Y. Furuya, Y. Kishi, M. Wuttig, Mater. Trans. 45, 204 (2004).

[16] R. Hassdorf, J. Feydt, S. Thienhaus, R. Borowski, M. Boese, T. Walther, M. Moske, MRS Online Proc. Library $\mathbf{7 8 5}$, 23 (2003).

[17] K. Oikawa, T. Ota, F. Gejima, T. Ohmori, R. Kainuma, K. Ishida, Mater. Trans. 42 , 2472 (2001). 
[18] Y. Qu, A. Gràcia-Condal, L. Mañosa, A. Planes, D. Cong, Z. Niec, Y. Ren, Y. Wang, Acta Mater. 177, 46 (2019).

[19] H. Chen, Yan-Dong Wang, Z. Nie et al., Nat. Mater. 19, 712 (2020).

[20] S.C. Lee, T.D. Lee, P. Blaha, K. Schwarz, J. Appl. Phys. 97, 10C307 (2005).

[21] P.J. Webster, K.R.A. Ziebeck, J. Phys. Chem. Solids 34, 1647 (1973).

[22] L. Bainsla, K.G. Suresh, Curr. Appl. Phys. 16, 68 (2016).

[23] E.I. Shreder, A.A. Makhnev, A.V. Lukoyanov, K.G. Suresh, Phys. Met. Metallogr. 118, 965 (2017).

[24] B. Venkateswarlu, P.V. Midhunlal, P.D. Babu, N. Harish Kumar, J. Magn. Magn. Mater. 407, 142 (2016).

[25] F. Ahmadian, A. Boochani, Physica B 406, 2865 (2011).

[26] D. Rani, K.G. Suresh, A. Alam, AIP Conf. Proc. 1953, 120007 (2018).

[27] L.Y. Wang, X.F. Dai, X.T. Wang, T.T. Lin, L. Chen, R. Liu, Y.T. Cui, G.D. Liu, Chin. Phys. B 24, 1 (2015).

[28] L.Y. Wang, X.T. Wang, L. Chen, Y. Zhang, Q. L. Xia, G.D. Liu, J. Supercond. Nov. Magn. 29, 995 (2016).

[29] P. Blaha, K. Schwarz, P. Sorantin, S.B. Trickey, Comput. Phys. Commun. 59, 399 (1990).

[30] J.P. Perdew, K. Burke, M. Ernzerhof, Phys. Rev. Lett. 77, 3865 (1996).

[31] A. Kokalj, Comput. Mater. Sci. 28, 155 (2003).

[32] H.J. Monkhorst, J.D. Pack, Phys. Rev. B 13, 5188 (1976).
[33] A. Dal Corso, Thermo_pw.

[34] P. Giannozzi, S. Baroni, N. Boniniet et al., J. Phys. Condens. Matter 21, 395502 (2009).

[35] F.D. Murnaghan, Proc. Natl. Acad. Sci. USA 30, 244 (1944).

[36] L. Vegard, Z. Phys. 5, 17 (1921).

[37] M. Born, K. Huang, Dynamical Theory of Crystal Lattices, Clarendon, Oxford 1956.

[38] S.R. Barman, A. Chakrabarti, S. Singh et al., Phys. Rev. B 78, 134406 (2008).

[39] A. Chakrabarti, M. Siewert, T. Roy, K. Mondal, A. Banerjee, M.E. Gruner, P. Entel, Phys. Rev. B 88, 174116 (2013).

[40] G.D. Liu, J.L. Chen, Z.H. Liu, X.F. Dai, G.H. Wu, Appl. Phys. Lett. 87, 262504 (2005).

[41] A. Tavana, L. Mikaeilzadeh, AIP Adv. 5, 117210 (2015).

[42] Q.M. Hu, C.M. Li, R. Yang, S.E. Kulkova, D.I. Bazhanov, B. Johansson, L. Vitos, Phys. Rev. B 79, 144112 (2009).

[43] C.M. Li, H.B. Luo, Q.M. Hu, R. Yang, B. Johansson, L. Vitos, Phys. Rev. B 82, 024201 (2010).

[44] H.B. Luo, C.M. Li, Q.M. Hu, R. Yang, B. Johansson, L. Vitos, Acta Mater. 59, 971 (2011).

[45] C. Tan, X. Tian, W. Cai, Physica B 404, 3662 (2009).

[46] S.V. Faleev, Y. Ferrante, J. Jeong, M.G. Samant, B. Jones, S.S.P. Parkin, Phys. Rev. Applied 7, 034022 (2017).

[47] J. H. Xu, T. Oguchi, Phys. Rev. B 35, 6940 (1987). 